FORMATION Formation emploi

Revue française de sciences sociales

131 | Juillet-Septembre 2015

Le Bac Pro a 30 ans

\title{
Les lycéens professionnels et la réforme du bac pro en trois ans : nouveau contexte, nouveaux parcours?
}

Which are the effects of the reform of the professional baccalaureat on the pathways of the vocational high schools pupils?

Französische Fachgymnasiasten und die Reform des Berufsabiturs

(Reduzierung auf 3 Jahre Unterricht) : neuer Kontext, neue Laufbahn?

Los alumnos de escuelas técnicas y la reforma del bachillerato técnico en tres años : ¿nuevo contexto, nuevos itinerarios?

Pierre-Yves Bernard et Vincent Troger

\section{OpenEdition}

Journals

Édition électronique

URL : http://journals.openedition.org/formationemploi/4447

DOI : 10.4000/formationemploi.4447

ISSN : 2107-0946

Éditeur

La Documentation française

Édition imprimée

Date de publication : 15 octobre 2015

Pagination : 23-40

ISSN : 0759-6340

Référence électronique

Pierre-Yves Bernard et Vincent Troger, «Les lycéens professionnels et la réforme du bac pro en trois ans : nouveau contexte, nouveaux parcours ? », Formation emploi [En ligne], 131 | Juillet-Septembre 2015, mis en ligne le 15 octobre 2015, consulté le 30 octobre 2020. URL : http://

journals.openedition.org/formationemploi/4447 ; DOI : https://doi.org/10.4000/formationemploi.4447

(c) Tous droits réservés 


\title{
Les lycéens professionnels et la réforme du bac pro en trois ans : nouveau contexte, nouveaux parcours?
}

\begin{abstract}
PierRe-YVEs Bernard Maître de conférences à l'université de Nantes. Chercheur au CREN (Centre de Recherches en Education de Nantes). Ses recherches portent sur les parcours scolaires et leurs relations avec l'insertion professionnelle, en particulier pour les jeunes se situant aux « marges » de la forme scolaire académique (jeunes en rupture scolaire, jeunes lycéens professionnels).

VINCENT TROGER Maître de conférences à l'ESPÉ (Ecole Supérieure du Professorat et de l'Education) de l'université de Nantes. Docteur en histoire de l'université de Paris 4 Sorbonne.

Chercheur au CREN (Centre de Recherches en Education de Nantes). Ses recherches et ses publications universitaires portent sur l'histoire et la sociologie de l'enseignement professionnel.
\end{abstract}

Résumé

Les lycéens professionnels et la réforme du bac pro en trois ans : nouveau contexte, nouveaux parcours?

Quels sont les effets de la réforme du baccalauréat professionnel sur les parcours des élèves de lycéens professionnels ? La réforme a accentué les intentions de poursuites d'études supérieures. Ces projets sont majoritairement orientés vers les sections de techniciens supérieurs. Mais la réforme crée aussi de nouveaux clivages selon les ressources sociales et la filière de formation. Certains intègrent avec succès des formations supérieures, d'autres alternent emplois précaires et formations plus ou moins qualifiantes. Cette recherche permet également de revisiter les modèles sociologiques consacrés à l'enseignement professionnel.

Mots clés : enseignement technique-professionnel, baccalauréat professionnel, politique de l'éducation, lycée professionnel, lycéen, cheminement scolaire, sociologie de l'éducation, poursuite d'études.

Abstract

Which are the effects of the reform of the professional baccalaureat on the pathways of the vocational high schools pupils?

The reform accentuated the intentions of pursuing in higher education. These intentions are mainly directed towards courses of technical studies. But the reform created also new splits according to the social resources and the training sector. Some students 
integrate higher education successfully, others alternate precarious employment and trainings more or less qualifying. This research also makes possible to revisit the main sociological models devoted to vocational education.

Keywords: technical \& vocational education, vocational baccalaureate, education policy, vocational upper secondary school, upper secondary school pupil, school paths, educational sociology, continuation of education.

Journal of Economic Literature: I 21

Traduction : Auteurs

En France, à la rentrée 2009, le baccalauréat professionnel en trois ans s'est substitué aux formations antérieures en quatre ans dans presque toutes les filières de l'enseignement professionnel.

Entre octobre 2009 et juin 2013, nous avons conduit une recherche sur un ensemble de lycées professionnels (LP) de l'académie de Nantes pour comprendre comment cette réforme était susceptible d'affecter les aspirations des élèves de la voie professionnelle (Bernard, Masy \&Troger, 2014). A partir de deux enquêtes par questionnaires et de 75 entretiens auprès des élèves, des enseignants et des chefs d'établissement, il s'agissait de saisir comment les élèves allaient donner du sens à leurs parcours dans ce nouveau cursus menant au bac pro.

Si nous utilisons ici la notion de parcours plutôt que celle de trajectoire, c'est parce qu'elle nous est apparue plus conforme aux conclusions que nous allons présenter ici. Alors que la notion de trajectoire renvoie à une analyse des choix d'orientation des élèves en termes d'enchaînement de déterminismes, la notion de parcours (Pollien, 2010) appréhende le cheminement et les choix d'orientation des élèves en termes d'interactions entre les déterminismes sociaux, les rationalités individuelles et les effets de contexte produits par l'évolution de l'offre de formation.

La première partie de ce texte est consacrée au rappel des principaux cadres d'analyse qui ont prévalu dans le champ de la sociologie de l'enseignement professionnel : celui de la sociologie de la reproduction et de ses héritages contemporains, (Palheta, 2012 ; Orange, 2010) ; celui d'une socio-histoire qui accorde aux contextes socio-économiques et à la rationalité des acteurs une dimension explicative plus déterminante que le précédent (Jellab, 2008); celui des conventions dans les régimes d'éducation et de formation qui situe dans un cadre sociétal plus global, les parcours scolaires et professionnels des acteurs (Verdier, 2001 ; Bernard \& Troger, 2013).

Les parties suivantes seront consacrées à la présentation de l'enquête conduite en deux temps, d'abord au cours des années scolaires 2009-2010 et 2010-2011, puis des années 2011-2012 et 2012-2013. Après avoir exposé la méthodologie suivie et les limites de 
cette recherche, nous insisterons sur ce qu'elle nous a permis de mettre en évidence à propos des aspirations des élèves de LP. La transformation de ces aspirations et leur confrontation avec la nouvelle offre de formation ont en effet produit de nouvelles formes de réussite et d'échec, avant et après le baccalauréat ; celles-ci participent à une évolution globale des parcours scolaires d'une proportion significative de la jeunesse française et incitent à revisiter les cadres théoriques existants.

\section{L'enseignement professionnel, objet d'analyses sociologiques divergentes}

Trois grandes familles d'analyse peuvent être identifiées dans le champ de la formation professionnelle initiale. La première est celle de la sociologie de la reproduction, héritière des approches de Pierre Bourdieu. La seconde s'inscrit, dans une certaine mesure, en réaction à la première, en proposant une analyse historiquement contextualisée des trajectoires des élèves de l'enseignement professionnel. La troisième analyse s'appuie sur une comparaison internationale pour comprendre les particularités du système français.

\subsection{Les analyses pionnières de la sociologie de la reproduction}

Les premiers travaux sociologiques consacrés à l'enseignement professionnel ont situé l'analyse des dispositifs d'éducation et de formation dans le cadre d'une interprétation en termes de rapports de domination.

Ils se sont inscrits dans la suite des "héritiers " de Bourdieu et ont analysé l'enseignement professionnel comme un des instruments de l'aliénation de la classe ouvrière, dont les enfants sont largement surreprésentés dans l'enseignement professionnel (Grignon, 1971 ; Charlot \& Figeat, 1985). L'ensemble de ces analyses suppose, d'une part, que les familles populaires subissent "l'imposition d'un ordre culturel légitime " (Delay, 2011) et, d'autre part, qu'elles intériorisent les verdicts scolaires stigmatisant leurs cultures, sans avoir conscience que ces verdicts sont le produit d'une domination symbolique.

Ugo Palheta (2012) mobilise les mêmes concepts dans un ouvrage récent : "Tout se passe donc comme si une fraction des jeunes d'origine populaire sécartaient d'eux-mêmes, par des décisions d'orientation peu ambitieuses, de cursus permettant d'accéder à des statuts sociaux plus valorisés et à des conditions d'existence moins précaires que celles statistiquement associées à une orientation vers l'enseignement professionnel » (p .66). 


\subsection{L'approche socio-historique et les stratégies d'acteurs}

Ugo Palheta nuance cependant son analyse en soulignant les clivages qui discriminent les publics de l'enseignement professionnels. Clivages entre sexes, entre apprentissage et formation scolarisée, entre niveau de diplôme préparé (CAP ou BEP - certificat d'aptitude professionnelle et brevet d'études professionnelles) ${ }^{1}$ et surtout entre spécialités enseignées. Ces clivages induisent un spectre de positions très différenciées dans le rapport à l'orientation des élèves de LP : à un pôle, on trouve ceux qui se projettent dans une carrière professionnelle correspondant à la spécialité qu'ils ont choisie et qui envisagent une poursuite d'études technologiques supérieures dans la même branche ; à l'autre pôle se situent ceux qui ne visent qu'à obtenir un diplôme dont ils espèrent qu'il leur permettra de se réorienter vers des études ou des activités professionnelles correspondant à leurs attentes.

Ugo Palheta rejoint ici les travaux d'auteurs qui ne s'inscrivent pas dans l'héritage de la sociologie de la reproduction, notamment ceux d'Aziz Jellab (2008). A partir des travaux d'histoire ou de socio-histoire (Tanguy, 1991 ; Brucy, 1998 ; Pelpel \& Troger, 2001 ; Troger, 2008), ce dernier rappelle que si l'enseignement professionnel a toujours accueilli les enfants des catégories populaires, il a accompli cette fonction dans deux contextes historiques distincts.

Depuis ses origines, dans les années 40, jusqu'au milieu des années 70 , il a bénéficié de la croissance des Trente Glorieuses, pour offrir à son public une opportunité de promotion socioprofessionnelle à travers un parcours qui n'était pas vécu dans un rapport d'infériorité vis-à-vis de l'enseignement général.

En revanche, à partir de la fin des années 70, dans un contexte de recul de l'emploi ouvrier, de croissance du chômage et de développement massif des diplômés dans la population active, l'enseignement professionnel n'a plus été en mesure de résister à la concurrence de l'enseignement secondaire général et est devenu majoritairement une filière de relégation pour les élèves en échec scolaire. Néanmoins, dans le présent numéro, Aziz Jellab montre que pour une fraction significative de ses élèves, l'enseignement professionnel demeure le lieu d'une réhabilitation scolaire leur permettant de redonner sens aux apprentissages. En partant d'un tout autre point de vue, celui des écarts de scolarisation entre filles et garçons en lycée professionnel, Séverine Depoilly (2014) montre que les parcours sexuellement différenciés ne sauraient se réduire au rapport de domination entre sexes ou aux dispositions incorporées. Ces dispositions socialement acquises interagissent avec l'offre de formation et le contexte économique pour délimiter un espace de décision au sein duquel filles et garçons construisent des parcours différenciés.

1. Ugo Palheta a effectué la recherche à l’origine de ce livre avant la réforme du bac pro en trois ans. 


\subsection{L'analyse en termes de régimes d'éducation et de formation}

Au-delà de ces deux premiers types d'approche, un autre cadre d'analyse peut être mobilisé ; c'est celui des régimes d'éducation et de formation, développé par Eric Verdier $(2000$; 2008) à partir des travaux initiaux de Maurice, Sellier et Sylvestre (1982) et de Boltanski et Thévenot (1991).

Il met en avant la notion de convention, définie comme un ensemble de principes de légitimité, qui fonde les opérations de jugement des acteurs et oriente leurs actions.

Dans le domaine scolaire, ce cadre d'analyse ne renvoie pas à un état des rapports sociaux ou économiques consubstantiel aux sociétés contemporaines, mais plutôt à un agencement institutionnel historiquement et spatialement situé, où la reconnaissance de la formation professionnelle dans le monde productif dispose d'une plus ou moins grande légitimité.

On peut identifier ainsi quatre types de conventions qui sont autant de formes de relations idéal-typiques entre école et société (Verdier, 2000 \& 2008 ; Bernard, 2009).

La première convention est dite académique, ou méritocratique. C'est la compétition dans le cadre scolaire qui permet d'atteindre la meilleure situation socio-professionnelle possible. La compétition méritocratique est au cœur de cette convention et dévalorise les savoirs pratiques.

A l'inverse, la deuxième conception est celle d'une école préparant à la vie active, selon une convention professionnelle. À ce titre, elle établit une relation de coopération entre l'éducation et la production, et valorise la dimension pratique des apprentissages, tant sur le plan des finalités (l'importance des savoirs appliqués) que sur celui des contextes (modèle de l'alternance, importance du «terrain »).

La troisième convention, dite universaliste, envisage l'école comme l'institution intégratrice par excellence. Elle vise à fonder une solidarité entre les membres de la société, à compenser les inégalités sociales et à fonder une démocratie effective par la transmission de valeurs et la création des conditions de l'égalité des chances.

La quatrième convention est dite marchande. Là où domine cette convention, l'activité scolaire est peu différenciée des autres activités professionnelles : libre choix des usagers, concurrence entre les établissements, standardisation des produits éducatifs à partir de normes de performance, transparence des résultats des établissements, financement de ceux-ci en fonction de la demande, voire financement privé.

En France, c'est la convention académique que Verdier présente comme dominante. La convention professionnelle n'est reconnue que dans l'enseignement professionnel ou l'apprentissage, c'est-à-dire dans les secteurs dominés du système éducatif.

Le bac pro peut être interprété comme une forme de compromis entre la convention professionnelle, en introduisant massivement les stages en entreprise dans les formations 
professionnelles scolaires, et la convention académique, en offrant aux meilleurs élèves de ces formations la possibilité de poursuivre des études supérieures (Verdier, 2001).

La réforme du baccalauréat professionnel en trois ans participe de ce mouvement, mais en introduisant explicitement l'embryon d'un principe universaliste, en égalisant la durée des cursus de bacheliers professionnels, généraux et technologiques.

Ces approches théoriques mettent donc chacune l'accent sur une dimension différente de l'analyse. Les approches sociologiques de la reproduction soulignent l'importance des structures sociales et leurs effets sur les élèves de milieux populaires, en montant comment leur déqualification par le système scolaire les amène à intérioriser un sentiment d'inaptitude aux études.

Symétriquement, l'approche socio-historique envisage cette intériorisation comme un espace de subjectivation dans lequel se construisent des choix, des préférences, etc. C'est la raison pour laquelle il nous semble indispensable de prendre au sérieux ce que nous disent les élèves de leurs aspirations et de leurs projets, même quand ceux-ci ressortent du registre de la passivité et de la frustration.

Pour autant, ce qu'ils disent de leur expérience scolaire s'appuie nécessairement sur les cadres institutionnels dans laquelle elle se déroule. A cet égard, la théorie des conventions éducatives nous semble fournir un modèle heuristique des principes mis en œuvre par les acteurs en situation.

\section{Les nouvelles ambitions des bacheliers professionnels}

Après avoir présenté la méthodologie de notre enquête, nous montrons que la réforme a significativement accru les poursuites d'études des bacheliers professionnels dans l'enseignement technologique supérieur, et dans quelles conditions ces poursuites d'études s'effectuent.

\subsection{Les enquêtes}

Notre enquête vise à comprendre comment les jeunes vivent leur parcours de formation, et quel sens (dans la double acception du terme : signification et orientation) ils lui confèrent.

Elle comporte deux volets :

Le premier volet est constitué de deux enquêtes quantitatives réalisées dans l'académie de Nantes, en seconde, puis trois ans plus tard, en terminale professionnelle. On ne peut toutefois pas parler d'un suivi de cohorte, les élèves interrogés en terminale pouvant par exemple provenir de formations de BEP ou de CAP. Quant aux élèves interrogés 
en seconde, certains ont interrompu leur formation avant la terminale, ou ont changé d'orientation, d'établissement, etc., et n'ont donc pas été interrogés une seconde fois. La première enquête a été conduite en octobre 2009, auprès d'élèves de seconde qui constituaient ainsi la première promotion d'élèves de bac pro 3 ans. Le questionnaire a été administré dans 16 établissements, permettant d'interroger 465 élèves. Le questionnaire portait sur les caractéristiques démographiques, sociales et scolaires des élèves, sur leur orientation en fin de troisième, sur leur satisfaction vis-à-vis de l'orientation engagée, et sur leurs aspirations quant à leur avenir. L'échantillon était assez représentatif du public national de l'enseignement professionnel en termes de répartition sexuée, d'âge et de répartition entre spécialités industrielles et tertiaires.

Une deuxième enquête quantitative a été réalisée en mai 2012, auprès de 532 élèves de terminale professionnelle inscrits dans 22 spécialités (sur 68 existantes), après l'enregistrement, par les élèves, de leurs vœux dans l'application "Admission post-bac ». Cette deuxième enquête a été menée dans les mêmes établissements et pour les mêmes spécialités. Le deuxième échantillon d'élèves de terminale se décompose en 416 élèves scolarisés dans le cursus de bac pro en trois ans, et 116 dans l'ancien cursus de bac pro en quatre ans.

Pour vérifier la validité de nos conclusions sur les effets de la réforme sur les parcours des élèves, il était en effet nécessaire de disposer d'éléments de comparaison avec les vœux exprimés et les orientations obtenues par les élèves de l'ancien cursus. L'échantillon est majoritairement masculin (56\%), dans une proportion quasi identique au niveau national (56,4\%, source DEPP, 2013). $31 \%$ de l'échantillon a 18 ans ou moins l'année du baccalauréat. 56,6\% des élèves interrogés sont enfants d'ouvriers ou d'employés (résultat obtenu à partir de la profession des pères, ou, à défaut, celle des mères), ce qui est très proche de la proportion nationale (54,3\%, source DEPP, 2012). La répartition des spécialités de formation de l'échantillon donne $54 \%$ en spécialités tertiaires, $46 \%$ en spécialités industrielles, ce qui là encore se rapproche fortement de la répartition nationale (56/44, source DEPP, 2012). Nous pouvons donc considérer l'échantillon comme suffisamment représentatif. Ce deuxième questionnaire avait pour but d'appréhender les aspirations des élèves de terminale professionnelle : poursuite d'études post-bac, ou entrée sur le marché du travail. À travers ce premier volet, nous pouvons donc saisir l'évolution des aspirations des élèves de la seconde à la terminale. À ce titre, il offre une certaine vision des parcours des élèves de LP.

Le second volet de cette recherche est constitué d'entretiens semi-directifs, afin de mieux comprendre les logiques d'action des acteurs impliqués dans la réforme du bac pro, élèves ou professionnels. Une première campagne d'entretien a été menée, à la fin de l'année scolaire 2009-2010, auprès de 22 élèves de seconde ayant participé à l'enquête par questionnaire, 11 de leurs enseignants, 7 chefs d'établissements et 3 cadres responsables de l'enseignement professionnel au niveau académique ; la seconde campagne, au cours du second semestre de l'année scolaire 2010-2011, auprès de 14 élèves du même échantillon, en classe de première, et de 20 enseignants des mêmes classes. 
À ces deux volets s'ajoutent des matériaux recueillis auprès des jeunes l'année suivant le baccalauréat, en 2012-2013 : un questionnaire auprès de 143 de ces jeunes, et 17 entretiens semi-directifs. Compte tenu du nombre restreint de personnes interrogées, il est inutile d'envisager une représentativité de ce groupe.

\subsection{Les aspirations des lycéens professionnels : un incontestable effet «bac pro en trois ans"}

Dès la classe de seconde, les élèves interrogés expriment une forte aspiration à la poursuite d'études post-baccalauréat. $59 \%$ de notre échantillon envisagent de poursuivre leur formation après le bac.

Ces élèves présentent certaines caractéristiques. Tout d'abord, ils se déclarent satisfaits ou plutôt satisfaits de leur orientation. Ce résultat est à souligner dans la mesure où il permet d'interpréter cette aspiration comme un prolongement d'une orientation assumée, plutôt que comme compensation d'une orientation subie.

Avoir une mère née à l'étranger augmente fortement l'aspiration à des études post-bac. Ce résultat confirme les travaux déjà réalisés sur les aspirations aux études des enfants d'immigrés, plus ambitieuses que celles des enfants d'origine française.

La situation d'emploi de la mère a également un effet très significatif. Les élèves dont la mère exerce un emploi envisagent plus fréquemment de poursuivre des études que ceux dont la mère est inactive ou au chômage. On peut voir là l'effet de sécurisation sur l'avenir qu'implique, pour le jeune, le fait d'avoir une mère exerçant effectivement un emploi.

Enfin, la classe suivie l'année précédente a un effet très significatif sur les perspectives des élèves : les aspirations aux études supérieures sont considérablement augmentées pour les élèves ayant déjà connu un début de second cycle, par rapport à ceux provenant de troisième à voie professionnelle.

Ces résultats appellent plusieurs remarques. L'aspiration aux études supérieures ne semble pas fortement liée aux caractéristiques scolaires, repérées ici par l'âge et le redoublement en primaire. Toutes choses égales par ailleurs, le milieu socioprofessionnel ne semble pas non plus jouer un grand rôle. On peut lire ces résultats comme le signe d'une généralisation des aspirations aux études supérieures dans tous les milieux sociaux, mais aussi de manière relativement indépendante du jugement académique de l'institution scolaire. Ils peuvent donc laisser présager des difficultés pour ces élèves à réaliser leurs aspirations, notamment en termes de compétences scolaires.

Ainsi, conçus depuis longtemps comme une filière de fin d'études dans une perspective d'insertion professionnelle immédiate, les LP deviendraient, au moins dans les représentations des élèves et de leurs familles, une autre forme d'accès à un certain type d'études supérieures (STS - section de technicien supérieur - essentiellement). 
Ce résultat est confirmé par la seconde enquête. $61 \%$ des élèves de terminale envisagent une formation après le bac et sont effectivement inscrits dans le logiciel « Admission Post $\mathrm{Bac} »$ de l'Éducation nationale.

Ce chiffre est nettement supérieur à celui qu'on enregistrait avant la réforme. Le taux d'inscription dans l'enseignement supérieur des bacheliers professionnels était de 23, $4 \%$ en 2008, à la veille de la généralisation du cursus en trois ans (DEPP, 2014). Ce taux ne comprend pas les inscriptions dans les formations en alternance, et sous-estime donc les poursuites de formation.

Si l'on s'en tient aux seuls vœux d'études à temps plein en formation supérieure, le chiffre de notre enquête est de 33,5\%. Compte tenu d'éventuelles non inscriptions et des échecs au bac, ce chiffre est assez cohérent avec la moyenne nationale de $28,7 \%$ cette année-là. Il y a donc un mouvement de croissance important de l'orientation des élèves de l'enseignement professionnel vers l'enseignement supérieur (voir graphique 1).

\section{Graph 1- Évolution du taux d'inscription des bacheliers professionnels dans I'enseignement supérieur (en \%)}

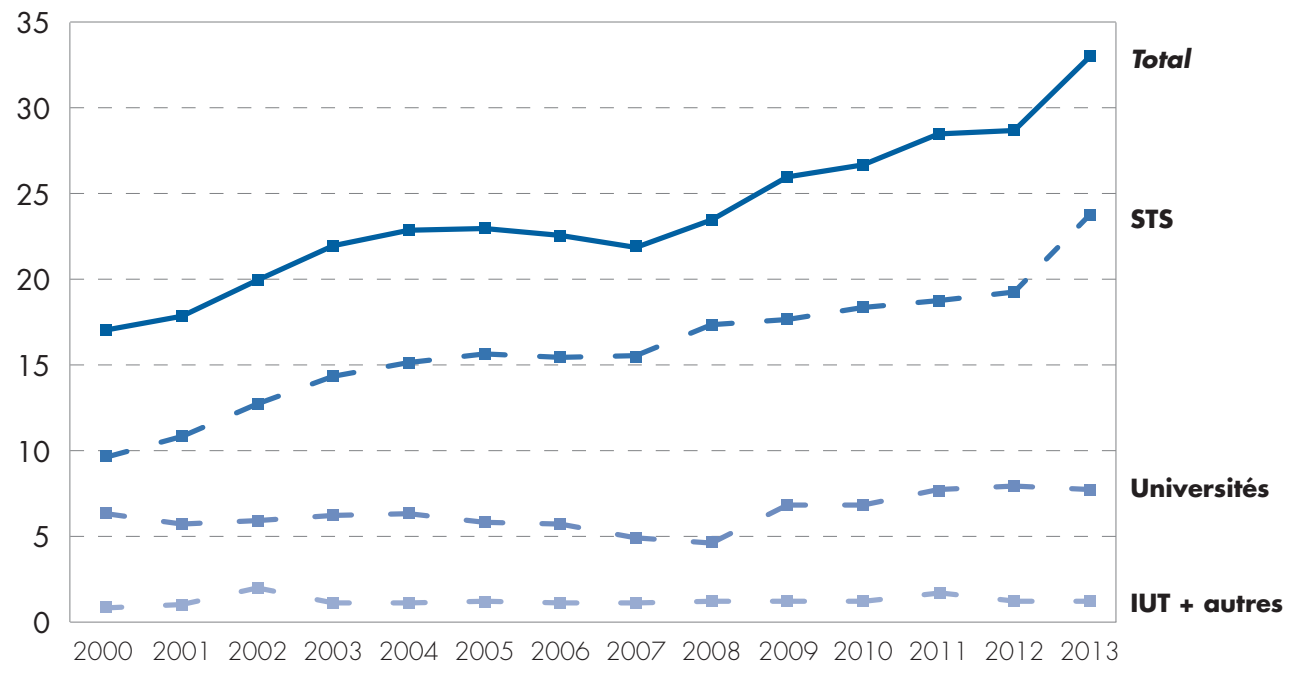

Sigles : IUT : Institut universitaire de technologie. STS : Sections de techniciens supérieurs. Exemple de lecture : en 2013, 23,8\% des bacheliers professionnels se sont inscrits en STS.

Source : DEPP, MENESR.

Toutefois, soulignons que la croissance des poursuites de formation de bacheliers professionnels avait commencé bien avant que les premières générations de bacheliers pro en trois ans aient terminé leur cursus. On ne peut donc pas imputer trop vite cette croissance à la seule réforme.

Les années 2000 ont été marquées par une forte croissance des taux d'inscription dans l'enseignement supérieur, grâce notamment à une plus grande ouverture des sections de 
techniciens supérieurs aux bacheliers professionnels, même si cette croissance semblait marquer le pas entre 2004 et 2008.

En tout état de cause, ces données conduisent à envisager l'hypothèse que la tendance haussière se serait poursuivie avec le maintien d'un bac pro en quatre ans. D'autant que le contexte économique peut aussi avoir provoqué une hausse des poursuites d'études. L'augmentation sensible du taux de chômage des jeunes diminue d'autant le coût d'opportunité de la poursuite des études, et favorise donc l'orientation des bacheliers professionnels vers l'enseignement supérieur.

Afin de tester l'hypothèse d'un effet de la réforme sur les aspirations à la poursuite d'études des élèves de lycée professionnel, nous avons bénéficié de l'expérience " naturelle " de la session 2012 du baccalauréat professionnel. Cette session offre en effet l'opportunité particulière de comparer, dans un même contexte, les élèves ayant suivi l'ancien cursus menant au bac pro en deux ans après un BEP et les élèves ayant suivi le nouveau cursus de bac pro en trois ans. La réforme du bac pro n'ayant été généralisée à l'ensemble des élèves de seconde qu'à la rentrée 2009, en 2009-2010, le BEP ancien régime a connu sa dernière promotion de seconde année. Il était ainsi possible d'interroger ces deux groupes d'élèves la même année, afin de comparer leurs vœux d'orientation.

Les données brutes de notre enquête montrent que le bac pro en trois ans augmente sensiblement les vœux d'orientation post baccalauréat $(65,5 \%)$ par rapport aux élèves ayant poursuivi un cursus de bac pro en quatre ans $(46,9 \%)$. Toutefois, compte tenu de la faible taille de l'échantillon, la probabilité que les deux groupes soient constitués d'individus aux caractéristiques différentes, donc non comparables, est élevée.

Pour réduire ce risque d'une comparaison biaisée, on a procédé à une modélisation « toutes choses égales par ailleurs " de l'orientation choisie après le bac, en prenant en compte les caractéristiques socio-économiques des élèves, la filière professionnelle choisie et les difficultés scolaires appréhendées à partir des éventuels redoublements (pour plus de précisions sur le modèle utilisé, voir Bernard, Masy et Troger, 2014). Nous avons choisi en particulier de neutraliser l'effet de la filière choisie. En effet les aspirations aux formations post-bac sont très inégales selon les spécialités, par exemple entre les spécialités du tertiaire administratif, où les trois quarts des élèves envisagent de poursuivre après le bac, alors qu'en chaudronnerie, plus de la moitié des élèves souhaitent travailler après avoir obtenu leur bac. Ces deux exemples ne sont pas significatifs d'une différence générale entre spécialités de services et spécialités de production. Parmi ces dernières, certaines formations semblent encourager la poursuite d'études (par exemple en électronique), alors que dans certaines formations tertiaires, les demandes de formations post-bac sont en dessous de la moyenne (restauration par exemple).

Pour raisonner toutes choses égales par ailleurs, nous avons décidé de regrouper les spécialités en trois groupes selon la part d'élèves sollicitant une formation post-bac : celles où la grande majorité des élèves (plus de $70 \%$ ) souhaitent poursuivre leurs études après 
le bac (systèmes électroniques numériques, comptabilité, secrétariat, vente, fabrication bois, métiers de la mode), celles où la majorité des élèves envisagent d'arrêter leurs études après le bac (maintenance véhicules automobiles, restauration, sécurité-prévention, technicien menuiserie, technicien chaudronnerie, maintenance des équipements énergétiques) et enfin celles où la poursuite d'études se situe dans la moyenne (commerce, esthétique, électrotechnique, maintenance des équipements industriels, usinage, accueil-relation clients, transport, agencement finition bâtiment, métallerie, techniciens froid et conditionnement).

Le modèle logistique ainsi réalisé montre que la réforme du bac pro en trois ans augmente significativement la demande de poursuite d'études supérieures des bacheliers professionnels, indépendamment des autres facteurs envisageables (tendance longue à la poursuite d'études, contexte économique) (voir tableau 1).

Tableau 1 : Modélisation d'une orientation « formation » (y compris en alternance) plutôt que « recherche d'emploi » à l'issue du bac pro

\begin{tabular}{|l|c|}
\hline $\begin{array}{l}\text { Parcours Bac Pro } 3 \text { ans } \\
\text { (Réf: parcours Bac Pro 2 ans après un BEP) }\end{array}$ & ns \\
\hline $\begin{array}{l}\text { Homme } \\
\text { (Réf: femme) }\end{array}$ & 0,639 \\
\hline $\begin{array}{l}\text { Milieu ouvrier/employé } \\
\text { (Réf: milieu autre) }\end{array}$ & 2,577 \\
\hline $\begin{array}{l}\text { Parents propriétaires du logement } \\
\text { (Réf: locataires) }\end{array}$ & 0,303 \\
\hline $\begin{array}{l}\text { Père né en France } \\
\text { (Réf: père né à l'étranger) }\end{array}$ & ns \\
\hline $\begin{array}{l}\text { Mère avec un diplôme inférieur au bac } \\
\text { (Réf: mère diplômée bac ou plus) }\end{array}$ & $0,612^{*}$ \\
\hline $\begin{array}{l}\text { Redoublement en primaire } \\
\text { (Réf: Pas de doublement en primaire) }\end{array}$ & 4,545 \\
\hline $\begin{array}{l}\text { Filières à parcours « mixtes » } \\
\text { Filières à parcours « supérieur » } \\
\text { (Réf: filières à parcours « emploi ») }\end{array}$ & 9,346 \\
\hline R2 (Nagelkerke) &, 242 \\
\hline
\end{tabular}

Note : Les chiffres indiqués sont des odd-ratios. Supérieur à 1, un odd ratio indique que la variable associée augmente la probabilité de choisir une orientation « formation » plutôt que « recherche d'emploi », et inversement. Le niveau de significativité statistique des odd-ratios est indiqué comme suit : Les coefficients indiqués sont significatifs au seuil de $5 \%$; sauf "* ", significatif à un seuil de risque compris entre $10 \%$ et $5 \%$. Les coefficients non significatifs (ns) ne sont pas indiqués. L'odd-ratio de 1,639 signifie que le parcours Bac pro 3 augmente fortement la probabilité de poursuivre en formation. Source : enquête réalisée par les auteurs auprès de 532 élèves de terminale professionnelle en 2012.

Ainsi, l'hypothèse d'une plus forte attractivité du bac pro en trois ans, fondée sur la plus grande ouverture de ce nouveau cursus vers les formations technologiques supérieures, est assez nettement confirmée par l'analyse.

Celle-ci révèle par ailleurs que cette transformation est inégale. Selon le modèle testé, les variables les plus déterminantes dans le choix de poursuivre après le bac sont la filière 
(les opportunités de poursuite sont très inégales selon les spécialités choisies), l’origine nationale (les enfants d'immigrés souhaitent plus souvent poursuivre leur formation) et la situation économique des parents, identifiée par la situation de propriétaire du logement : plus cette situation est favorable, plus les élèves envisagent une poursuite de formation.

Ce dernier point montre que, même si les élèves du lycée pro sont issus très majoritairement de milieux populaires, les inégalités de conditions de vie enregistrées dans ces milieux ont un impact important sur les anticipations des familles et des jeunes.

\section{Entre revalorisation 3 et nouvelles inégalités}

Les résultats que nous venons d'exposer permettent d'interroger les analyses antérieures en montrant que la réforme du baccalauréat professionnel a ouvert la possibilité d'une transformation significative de la place et du rôle de l'enseignement professionnel dans le dispositif français d'études post-obligatoires.

\subsection{Un nouvel espace pour les stratégies d'acteurs ...}

Au-delà de ces constats, cette transformation des aspirations n'aligne pas les projets des élèves de l'enseignement professionnel sur le modèle des parcours académiques de l'enseignement général.

Les élèves que nous avons interrogés dans le cadre des entretiens manifestent, à cet égard, une profonde dualité, entre, d'une part, l'aspiration aux études et, d'autre part, une forme plus ou moins marquée de rejet de l'école.

Cette dualité s'exprime unanimement chez tous les élèves que nous avons interrogés, quels qu'aient été leurs résultats antérieurs au collège. Tous expriment une forme de lassitude qui porte autant sur le sens de la scolarité ("J'en avais marre des cours généraux, ça se passait mal quoi. En fait, je me levais le matin pour me dire: "mais en fait, qu'est-ce que je fous en cours?" "), que sur les contraintes de la forme scolaire ("C'est pas que ça me dérange, mais rester assis toute une journée, je ne peux pas. (...) Ouais, écouter quelqu'un parler pendant huit heures, je ne peux pas, je pouvais pas») (Bernard, Delavaux et Troger, 2011).

Mais simultanément, ils s'inscrivent dans un modèle contemporain de la jeunesse, où le parcours se construit au gré des expériences. Même s'ils attribuent la décision de poursuivre des études plus souvent à leur motivation qu'à leurs résultats scolaires, leur position dominée dans la hiérarchie scolaire les place dans une forte situation d'incertitude, où l'éventualité d'une poursuite d'études se construit en fonction d'un mélange d'opportunités, de motivation et de sentiment de compétences perçues. Ainsi, un jeune lycéen interrogé en seconde considère-t-il sa scolarité au lycée comme une phase 
de réflexion sur ce qu'il fera de sa formation en bac pro : "Ben, je sais pas vraiment si je continue ou si je travaille, je sais vraiment pas, bon, j'ai encore deux ans, donc j'ai le temps de réfléchir quand même."

Toutefois, malgré cette incertitude, les lycéens interrogés sont persuadés de l'utilité d'une formation la plus longue possible, comme le déclare simplement l'un d'entre eux : "Oui, plus y'a d'études, mieux c'est après."

Ils éprouvent également le sentiment de se rapprocher d'un modèle commun, en accédant à une forme d'égalité symbolique que constitue la même durée d'études pour préparer un baccalauréat : "alors, le bac pro, on est tous égaux, on a tous le même parcours, on va tous avoir le bac la même année et après on pourra faire les mêmes études."

Cette tension entre désir de formation et rejet de l'école traduit d'abord une forme de compromis entre des aspirations familiales fortes et un jugement scolaire peu valorisant, tout au moins jusqu'au collège. En effet, on assiste à une forte convergence des aspirations des familles de tous milieux sociaux, y compris dans les milieux populaires, vers la nécessité du diplôme comme protection contre les risques d'exclusion sociale (Poullaouec, 2010). On évoluerait ainsi vers une orientation plus souvent assumée dans le cadre de stratégies "de détour" (Charlot, 1997), que nous appellerions plutôt de " contournement ", pour accéder au baccalauréat et aux poursuites d'études postbaccalauréat sans avoir à subir la contrainte de l'enseignement général.

Il y a donc un premier niveau de compromis qui se situe entre l'élève et sa famille : "J'aurais préféré faire un BEP mais bon, voilà, donc j'ai fait un bac pro, parce que ma mère elle voulait que je fasse un bac pro, pas un CAP."

Soulignons que ce premier niveau peut s'étioler avec le temps, notamment quand la formation professionnelle n'offre pas un espace de motivation et d'ambition au jeune : "Eux, [les parents] ils voulaient que je fasse un bac pro et après, voire pour continuer plus tard. Pour le salaire. Même encore, ils me disent de continuer après, mais j'en ai marre" nous déclare un jeune lycéen professionnel en classe de première.

Un deuxième niveau de compromis se situe dans l'organisation même de la formation professionnelle. Poursuivre des études est envisageable, à condition que la formation laisse une large place à la dimension " pratique " : "Les études, c'est pas trop mon truc (...) faut que je trouve un truc qui me plaise pour que je sois motivé après. Et puis à faire en alternance, parce que sinon je pense que je pourrais pas. "Cette revendication d'un rapport au savoir pratique peut même être vécue comme une forme de légitimité concurrente de la formation académique générale, jugée abstraite et déconnectée du monde du travail : "Et puis des fois, ça me fait bien rire des fois, le bac général, parce que nous on se fait prendre pour des branquignoles parce qu'on est en professionnel, mais ceux qui ressortent du bac général, ils ont pas de boulot, donc voilà." 
Les références à la pratique, aux savoirs professionnels, au monde du travail se construisent pendant la scolarité en lycée professionnel, dans les interactions qui se nouent entre élèves, entre élèves et enseignants, et à travers les premiers contacts avec le monde du travail. Cette construction affecte fortement les aspirations de poursuite de formation au fur et à mesure du déroulement de la scolarité. Alors qu'en classe de seconde, les entretiens révèlent des ambitions d'études supérieures fondées sur la recherche d'un meilleur niveau, sans se préoccuper de la spécialité de formation, celle-ci structure fortement les vœux des élèves en terminale. Dans l'enquête réalisée en 2012, la majorité des projets de poursuite de formation concerne la même spécialité que celle qui a été suivie au lycée. De fait, ce sont les sections de techniciens supérieurs qui constituent le débouché le plus fréquemment souhaité après un bac pro.

Cette construction d'aspirations fondées sur des principes de qualification professionnelle peut être interprétée comme l'adhésion à la convention professionnelle au sens de Verdier (Bernard \& Troger, 2013). A ce titre, nombre d'élèves confèrent à leur expérience scolaire le sens d'une formation au métier, transposable dans le monde du travail et valorisant la dimension pratique des apprentissages. Quand ils entrent en STS, ces élèves revendiquent cette qualité, notamment par comparaison avec les élèves issus de l'enseignement général : "Mais là aujourd'hui, je me dis que c'est vraiment un super atout [le bac pro], parce que là, tu vois, y'a un mec qui sort de BTS, tu lui dis 'vas-y" et bah il sait pas. Tandis que moi, direct. Donc, moi je pense que c'est vraiment un super atout au final."

De ce point de vue, l'effet d'attraction produit par la réforme en raison de l'égalité symbolique avec les filières générales et technologiques ne s'accompagne pas, ou seulement de manière très marginale, d'un effet centrifuge qui augmenterait les tentatives de poursuite d'études générales. Au contraire, c'est la voie technologique supérieure, et essentiellement les BTS, que les élèves de bac pro 3 ans semblent envisager comme un débouché " naturel " de leur formation, de manière encore plus prononcée qu'au moment de leur entrée dans la filière.

\section{2 ... mais un " vocationnalisme " restreint}

Toutefois, cette légitimation a posteriori d'une orientation en enseignement professionnel se heurte à un certain nombre de difficultés une fois le bac passé. Ce sont tout d'abord les difficultés liées aux faibles compétences académiques qui placent les étudiants issus du bac pro en situation d'infériorité par rapport aux autres bacheliers : "Nous, on a vraiment appris que les bases, et ça suffit pas du tout pour faire ce genre de BTS. Les bases, elles sont pas assez approfondies. "

Les jeunes bacheliers professionnels doivent aussi affronter la réalité de leurs habitudes de travail, largement inférieures à ce qui est attendu d'eux en BTS : "Et l'autre problème, c'est le temps à consacrer, que moi je n'avais pas. Dans le sens où moi j'ai fait un bac 
pro. Le bac pro, c'est pas pour le martyriser ni rien, mais on fait rien en fait. Enfin si, c'est quelque chose, mais on n'a pas de devoirs, on n'est pas habitué au travail à la maison."

Pour beaucoup de ceux que nous avons interrogés, le parcours post-bac se transforme en une alternance d'échecs en formation, d'emplois précaires, de reprises de formation, au cours desquels ils tentent de mobiliser les compétences qu'ils pensent avoir acquises et d'en acquérir de nouvelles.

Les publics que nous avons interrogés se clivent alors en fonction, d'une part, des ressources matérielles et psychologiques qu'ils sont susceptibles de mobiliser pour saisir les opportunités qui s'offrent éventuellement à eux, et, d'autre part, de l'état du marché du travail dans la spécialité dans laquelle ils se sont formés.

Les clivages entre spécialités émergent ainsi à nouveau avec force. Dans les spécialités industrielles, où les effectifs sont faibles et les débouchés souvent encore significatifs, les bacheliers professionnels y sont nombreux, parfois majoritaires, et leur expérience pratique acquise en stages peut constituer un différentiel favorable vis-à-vis des élèves issus des filières technologiques : "Parce que nous, dans l'entreprise, on fait du dessin mais aussi on fait de la machine. (...) Et moi, je pense que c'est aussi pour ça que j’ai été pris, parce que comme j'ai un CAP, je sais, en gros, tourner les manivelles. " Pour ces jeunes diplômés, la réussite au bac constitue clairement une étape décisive qui les inscrit dans une dynamique de réussite ou, comme le note Aziz Jellab (2014), dans un processus d'émancipation scolaire: "Je sais qu'après on peut faire une licence pro ou une licence. Après... Après il y a les écoles, ou les écoles d'ingénieurs. "

A l'opposé, dans les spécialités où le marché de l'emploi est difficile et où les effectifs sont souvent chargés, particulièrement dans les formations du secteur tertiaire, les bacheliers professionnels, minoritaires, décrochent en nombre et éprouvent alors la réalité de la faible reconnaissance des diplômes professionnels :

Enquêteur : "Ça veut dire que le BTS ne t’apportait rien de plus ? Même sur le marché de l'emploi?"

"Bah non... Déjà, ça leur est un peu égal qu'on ait un bac pro ou juste un BEP. Ce qu'ils veulent eux, c'est la... ouais, ce qu'ils veulent, c'est qu'on ait déjà travaillé. "

Ces décrochages du supérieur renforcent ainsi les déterminants sociaux des parcours de formation. Face aux désillusions des expériences de l'enseignement supérieur, l'entrée dans un monde du travail précarisé s'opère dans une résignation désabusée, comme pour cette bachelière professionnelle en comptabilité, après une expérience malheureuse en BTS : "Mon père m'a demandé d'aller chercher du travail. Après, je sais pas, parce que je me dis que... Y'a beaucoup de gens qui nous disent après le bac "vous aurez rien, vous trouverez jamais rien. Le monde du travail est trop compliqué. Il faut continuer les études". Donc, moi, dans ma tête, eh ben, c'est dans cette logique là que je partais, donc voilà. Mais sinon... Ah oui, je vais chercher du travail, j'ai déjà un emploi étudiant. " 
Cet extrait illustre bien une des limites fondamentales du "vocationnalisme à la française " (Verdier, 2010) : à la différence des régimes d'éducation et de formation " germaniques» (Allemagne, Autriche, Suisse, ...), on n’y reconnait pas de véritable institutionnalisation de la relation formation-emploi à travers des normes solidement établies par les partenaires sociaux ; cela restreint d'autant les possibilités de réussite des nouveaux parcours post-baccalauréat que la réforme offre pourtant aux bacheliers professionnels et dont une majorité d'entre eux tente de se saisir.

\section{Conclusion}

Notre étude éclaire les effets de la réforme du baccalauréat professionnel en trois ans, tant sur les aspirations des lycéens que sur la réalité des parcours post-baccalauréat.

Dés l'entrée en seconde, une forte majorité d'entre eux envisage explicitement de poursuivre sa formation après la terminale, tout en manifestant une inappétence pour les formes scolaires académiques. Ces intentions se concrétisent, après le baccalauréat, par un taux significatif d'inscriptions dans l'enseignement supérieur technologique court, notamment dans les formations par alternance.

Soulignons néanmoins que ces aspirations sont, dès l'origine, inégalement réparties. Les parcours scolaires antérieurs et les conditions socio-économiques familiales clivent les ambitions, tant en termes de choix de spécialités que de projets de poursuites de formation.

De la même manière, la réussite des parcours dans l'enseignement supérieur technologique court est difficile pour la majorité des bacheliers professionnels ; elle est en effet fortement corrélée aux ressources que les élèves sont capables de mobiliser et à la configuration des spécialités qu'ils choisissent.

En outre, en écartant les formations de CAP, notre enquête sous-estime sans doute la proportion des élèves qui renoncent à affronter un parcours qui n'est plus balisé à mi-chemin par le BEP. De même, nous n'avons pu mesurer avec certitude, à l'échelle de notre enquête, les effets de la réforme sur les abandons en cours de formation. Une enquête de la DEPP révèle néanmoins que le raccourcissement à trois ans de la durée de la préparation au bac pro n’a pas augmenté le taux de décrochage en cours de formation, au contraire (Defresne et Dubois, 2013).

Comme ultime limite à ce travail, rappelons que notre enquête a été conduite dans une région, les Pays de la Loire, qui se caractérise par une moindre dévalorisation de l'enseignement professionnel dans les choix d'orientation des familles.

Au terme de cette recherche, il semble qu'il faille envisager les parcours scolaires comme le produit d'une interaction permanente entre trois champs autonomes : celui des logiques internes de l'institution et de l'offre de formation qu'elle constitue, celui des structures socio-économiques en tant qu'elles déterminent les frontières d'un espace de choix et d'action propre à chaque catégorie ou groupe sociaux, et celui enfin des acteurs 
et de leurs propres logiques d'action, dont nous considérons qu'elles renvoient à des valeurs partagées, des représentations et des expériences individuelles ou collectives, telles qu'en rend compte le modèle des conventions. Cette approche permet de prendre en compte les contextes spatiaux et temporels tout en intégrant le poids des déterminismes sociaux, culturels et économiques.

\section{Bibliographie}

Bernard P.-Y. (2009), La politique de traitement du décrochage scolaire. Le cas de la Mission générale d'insertion de l'Education nationale, thèse de doctorat, université de Nantes.

Bernard P.-Y., Delavaud L. \& Troger V. (2011), Le baccalauréat professionnel en trois ans : perspectives et risques pour les lycéens, rapport de recherche pour la Direction de l'évaluation de la prospective et de la performance, CREN, MSH.

Bernard P.-Y. \& Troger V. (2013), " La réforme du bac professionnel en trois ans : vers un renforcement de la convention professionnelle dans le système éducatif français ? ", L'orientation scolaire et professionnelle, 42(2), pp. 273-297.

Bernard P.-Y., Masy J. \& Troger V. (2014), Le baccalauréat professionnel en trois ans : les élèves de lycée professionnel entre nouvelles trajectoires de promotion scolaire et risques d'espoirs déçus, CREN, rapport de recherche pour le centre Henri Aigueperse/UNSA, 81 pages, http:// cha.unsa-education.com/spip.php?article72.

Boltanski L. \& Thévenot L. (1991), De la justification. Les économies de la grandeur. Gallimard, Paris.

Brucy G. (1998), Histoire des diplômes de l'enseignement technique et professionnel (18801965). L'Etat, l'école et les entreprises et la certification des compétences, Belin, Paris.

Charlot B. (1997), Du rapport au savoir, Anthropos, Paris.

Charlot B. \& Figeat M. (1985), Histoire de la formation des ouvriers, Minerve, Paris.

Defresne F. \& Dubois M. (2013), «Le baccalauréat professionnel en trois ans après la troisième : vers une élévation du niveau des élèves? ", Education et formations, 84, pp. 61-69.

Delay C. (2011), Les classes populaires à l'école. La rencontre ambivalente entre deux cultures de légitimités inégales, Presses Universitaires de Rennes, Rennes.

DEPP (2012), Repères et références statistiques, Direction de l'évaluation, de la performance et de la prospective, Paris.

DEPP (2013), Repères et références statistiques, Direction de l'évaluation, de la performance et de la prospective, Paris.

DEPP (2014), Repères et références statistiques, Direction de l'évaluation, de la performance et de la prospective, Paris. 
Depoilly S. (2014), Filles et garçons au lycée pro, Presses universitaires de Rennes, Rennes.

Grignon C. (1971), L'ordre des choses. Les fonctions sociales de l'enseignement technique, Éditions de Minuit, Paris.

Jellab A. (2008), Sociologie de l'enseignement professionnel, Presse Universitaires du Mirail, Toulouse.

Jellab A. (2014), L'émancipation scolaire. Pour un lycée professionnel de la réussite, Presse Universitaires du Mirail, Toulouse.

Jellab A. (2015), "Apprendre un métier ou poursuivre ses études ? Les élèves de lycée professionnel face à la réforme du bac pro trois ans ", Formation Emploi, 131, juillet-septembre, La Documentation française.

Maurice, M., Sellier, F. \& Silvestre J.-J. (1982), Politique d'éducation et organisation industrielle en France et en Allemagne. PUF, Paris.

Orange S. (2010), "Le choix du BTS ", Actes de la recherche en sciences sociales, 183, pp. 32-47.

Palheta U. (2012), La domination scolaire. Sociologie de l'enseignement professionnel et de son public, PU, Paris.

Pelpel P. \& Troger V. (2001), Histoire de l'enseignement technique, L'Harmattan, Paris.

Poullaouec T. (2010), Le diplôme arme des faibles, La Dispute, Paris.

Pollien A. (2010), «Faire une pause ou bifurquer, essai de typologie des trajectoires de formation ", Education et société 26, pp. 123-143.

Tanguy L. (1991), L'enseignement professionnel en France. Des ouvriers aux techniciens, PUF, Paris.

Troger V. (2008), "Les enseignements techniques et professionnels , in Van Zanten A. (Ed.), Dictionnaire de l'éducation, PUF, Paris.

Verdier E. (2000), «Analyse sociétale et changement institutionnel : le cas de l'éducation et de la formation professionnelle initiale ", in Tallard M., Théret B. \& Uri D. (dir.) Innovations institutionnelles et territoires, L'Harmattan, Paris, pp. 101-128.

Verdier E. (2001), " La France a-t-elle changé de régime d'éducation et de formation ? », Formation Emploi, 76, pp. 11-34.

Verdier E. (2008), «L'éducation et la formation tout au long de la vie : une orientation européenne, des régimes d'action publique et des modèles nationaux en évolution ", Sociologie et Sociétés, 40 (1), pp. 195-225.

Verdier E. (2010), « Postface : L'orientation scolaire et professionnelle : entre assignation et idées floues, l'anarchie organisée ", Formation Emploi, 109, pp. 113-126. 